\title{
ATIVIDADES DE DIVULGAÇÃO CIENTÍFICA SOBRE EXOPLANETAS
}

\section{SCIENTIFIC DISSEMINATION ACTIVITIES ON EXOPLANETS}

\author{
Ryan Nepomuceno Montemor ${ }^{1}$ \\ Ricardo Roberto Plaza Teixeira ${ }^{2}$
}

\begin{abstract}
Resumo: Este trabalho investigou os resultados e impactos de atividades de divulgação científica sobre a ciência dos exoplanetas, ocorridas em 2018, junto a alunos de quatro escolas de educação básica situadas em municípios do litoral norte paulista. Essas ações permitiram que os alunos adquirissem melhor compreensão acerca dos conceitos apresentados sobre exoplanetas e procuraram incentivar o aprofundamento no estudo de áreas da astronomia. Para a elaboração das apresentações estudou-se tanto o estado atual dos conhecimentos científicos sobre exoplanetas, quanto o modo como o trabalho didático com temas de astronomia pode ser efetuado a alunos dos ensinos fundamental e médio. Nas apresentações foram usados diferentes recursos que procuraram tornar mais compreensíveis os conceitos científicos envolvidos e as técnicas de detecção de exoplanetas. Em particular, considerou-se o caráter interdisciplinar da ciência dos exoplanetas, bem como, conhecimentos relacionados à história da astronomia.
\end{abstract}

Palavras-chave: Astronomia; Ensino de Física; Divulgação da Ciência; Detecção de Exoplanetas.

\begin{abstract}
This work investigated the results and impacts of scientific dissemination activities on the science of exoplanets, which took place in 2018, with students from four schools of basic education located in municipalities on the north coast of São Paulo. These actions allowed students to gain a better understanding of the concepts presented about exoplanets and sought to encourage further study in areas of astronomy. For the elaboration of the presentations, it was studied both the current state of the existing scientific knowledge about exoplanets, as well as the way the didactic work with astronomy themes can be carried out with elementary and high school students. In the presentations, different resources were used to make the scientific concepts involved and the techniques for detecting exoplanets more understandable. In particular, the presentations considered the interdisciplinary nature of the science of exoplanets, as well as knowledge related to the history of astronomy.
\end{abstract}

Keywords: Astronomy; Physics Teaching; Dissemination of Science; Exoplanet Detection.

\section{Introdução}

Este é um artigo que investiga as possibilidades educacionais da inserção de conteúdos relacionados ao estudo dos exoplanetas em atividades de divulgação científica. Durante as ações desenvolvidas no âmbito desta pesquisa ficou evidente a importância de uma abordagem interdisciplinar na educação científica, sobretudo para a aprendizagem

\footnotetext{
${ }^{1}$ Graduanda do curso de Licenciatura em Física do Instituto Federal de São Paulo (IFSP), campus Caraguatatuba, São Paulo, Brasil. E-mail: montemor.n@aluno.ifsp.edu.br.

${ }^{2}$ Doutor em Física pela Universidade de São Paulo (USP). Docente do Instituto Federal de São Paulo (IFSP), campus Caraguatatuba, São Paulo, Brasil. E-mail: rteixeira@ifsp.edu.br.
} 


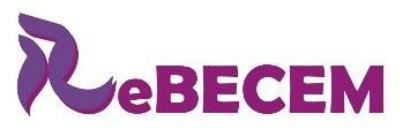

Revista Brasileira de Educação em

Ciências e Educação Matemática

DOI: https://doi.org/10.33238/ReBECEM.2021.v.5.n.2.26865

de temas localizados próximos à fronteira da ciência atual, como foi o caso. Ao longo deste trabalho foram analisados os resultados de atividades de divulgação científica sobre exoplanetas, ocorridas no segundo semestre de 2018, junto a alunos de quatro instituições escolares públicas de Educação Básica situadas em municípios do litoral norte do estado de São Paulo; duas dessas apresentações aconteceram para estudantes do ensino médio e as outras duas, para estudantes dos dois últimos anos do ensino fundamental.

É importante que a aprendizagem da Física dialogue com as descobertas científicas mais recentes e com os avanços tecnológico, pois isso colabora para despertar o interesse dos alunos e ajuda a superar a ideia equivocada de que a aprendizagem dessa ciência deve ocorrer apenas por meio de memorização de fórmulas. A desproporcional ênfase conferida às operações e técnicas matemáticas usadas para resolver problemas abstratos e descontextualizados, muitas vezes, acaba deixando de lado a compreensão mais efetiva dos conceitos físicos (ANDRADE, 2012).

A ciência de forma geral - e a Física, em particular - é um modo de olhar para o mundo e uma forma de questionar a natureza das coisas, pela leitura crítica e atenta acerca de experiências e observações realizadas (MANDELL, 2013). Em específico, o estudo de tópicos da área da Astronomia pode efetivamente colaborar para o ensino de Física ao tornar a sua aprendizagem mais interdisciplinar.

\section{A ciência dos exoplanetas}

O sistema solar possui oito planetas e, dentre eles, até o momento, apenas no planeta Terra foi constatada a existência de vida como a conhecemos (NASA, 2006). Há séculos, muitos pesquisadores especulam a respeito da possibilidade de existência de astros pertencentes a sistemas planetários distintos do nosso, os exoplanetas. Giordano Bruno - importante astrônomo italiano do século XVI, executado pela inquisição da Igreja Católica no ano de 1600, devido às suas ideias heliocêntricas - sugeriu, em 1584, a existência de exoplanetas em sua obra "Sobre o infinito, o universo e os mundos" (BRUNO, 1978, p. 44) quando no diálogo terceiro, o protagonista Elpino afirma textualmente: "Existem, pois, inúmeros sóis, existem terras infinitas, as quais se movimentam à volta daqueles sóis [...] Nós podemos ver os sóis que são os maiores, antes grandíssimos corpos, mas não podemos perceber as terras, as quais, por serem corpos muito menores, são invisíveis [...]”. Mais de 400 anos após sua morte, a hipótese teórica 


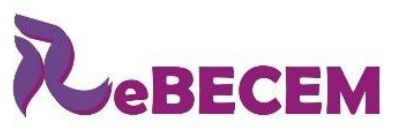

Revista Brasileira de Educação em

Ciências e Educação Matemática

DOI: https://doi.org/10.33238/ReBECEM.2021.v.5.n.2.26865

original de Giordano Bruno de que planetas como a Terra poderiam existir em outras partes do universo evoluiu. Atualmente, na astrofísica, existe a determinação experimental de milhares de exoplanetas, ou seja, de sistemas planetários extra-solares.

Em outubro de 1995, os astrônomos suíços Michel Mayor e Didier Queloz, em uma conferência na Itália, relataram a detecção do primeiro exoplaneta circulando uma estrela semelhante ao Sol, por meio de medidas da velocidade radial da estrela em que ele orbita (MAYOR; QUELOZ, 1995). Os dados foram obtidos pelo Observatório de Haute-Provence, situado na região sudeste da França. Esse exoplaneta denominado "51 Pegasi B" (ou, simplesmente, "51 Peg") e situado a aproximadamente 51 anos-luz do sistema solar, pertence a uma classe planetária chamada de "Júpiteres Quentes", que inclui uma parcela considerável dos exoplanetas já encontrados; o que caracteriza esta classe é tanto a sua massa ser grande como a massa de Júpiter, quanto a sua distância até a estrela em torno da qual orbita ser bem inferior à distância da Terra ao Sol, conferindolhes altas temperaturas (AMORIM; SANTOS, 2017).

As características inusitadas de "51 Peg", como ter um raio orbital cerca de 5\% do da Terra, uma órbita de aproximadamente 4,2 dias e uma massa de 150 massas terrestres (da ordem de grandeza da massa de Júpiter que é de cerca de 318 massas terrestres) surpreenderam muitos pesquisadores que pressupunham, até então, que planetas massivos deveriam estar localizados longe de suas estrelas-mães (FISCHER et al., 2010). No ano de 2019, Mayor e Queloz foram laureados com o Prêmio Nobel de Física pela descoberta pioneira de um exoplaneta orbitando uma estrela do tipo solar que eles fizeram nos anos 1990.

A partir desse momento em que as "comportas" foram abertas, iniciou-se uma vigorosa e crescente expansão do número de exoplanetas conhecidos. A descoberta de “51 Peg”, seguiu-se à sugestão feita anteriormente por Otto Struve (1952) de que um planeta com dez vezes a massa de Júpiter seria fácil de detectar, visto que que faria com que a velocidade radial observada da estrela oscilaria em cerca de $2 \mathrm{~km} / \mathrm{s}$. Apesar de " 51 Peg" ser considerado o primeiro exoplaneta descoberto orbitando uma estrela semelhante ao Sol, é importante notar que esse não foi o primeiro exoplaneta a ser encontrado orbitando qualquer categoria de estrela (BANKS; RHODES; BUDDING, 2020), pois alguns anos antes, sinais de rádio de um pulsar forneceram evidências convincentes sobre a existência de massa planetária fora do nosso sistema solar, mas nesse caso, orbitando uma estrela de nêutrons (WOLSZCZAN; FRAIL, 1992). 

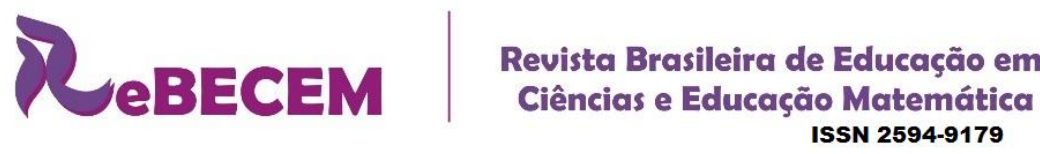

DOI: https://doi.org/10.33238/ReBECEM.2021.v.5.n.2.26865

Existem centenas de bilhões de galáxias no universo e uma galáxia como a Via Láctea possui algo da ordem de centenas de bilhões de estrelas, portanto, possivelmente há no universo uma enorme quantidade de sistemas planetários. Epicuro, por volta de 300 a.C., em carta a Heródoto, já especulava que deveria existir um número infinito de mundos, tanto semelhantes ao nosso mundo, quanto diferentes (PERRYMAN, 2018). Por consequência, deve existir uma grande variedade de planetas com distintas características, tais como massa, raio (tamanho), período orbital, temperatura, distância até a estrela em torno da qual orbita, energia disponível, composição química e, em específico, a capacidade de manter a água no estado líquido, uma condição para a existência de vida da forma como conhecemos. Tais dados são de suma importância para a análise, pois com os resultados obtidos, é possível indicar o índice de habitabilidade que cada exoplaneta possui (ALMEIDA, 2017). Esse novo campo da astronomia, a exoplanetologia ou ciência exoplanetária produz dois tipos de atividades principais: (i) detecção de novos planetas; (ii) estudos para compreender os processos físicos e dinâmicos de planetas individuais, dos sistemas planetários e das interações dos planetas com suas estrelas hospedeiras (SCHNEIDER et al., 2011).

Uma das fontes de informações sobre exoplanetas já conhecidos é o "Arquivo de Exoplanetas da NASA"3 ("NASA Exoplanet Archive"), que apresenta dados úteis para atividades de ensino e de divulgação científica acerca deste tema (AKESON et al., 2013). Dentre as ferramentas disponibilizadas por essa plataforma, estão tabelas interativas com informações a respeito de exoplanetas, cuja existência foi confirmada, inclusive com o recurso de filtrar e selecionar dados. Essa ferramenta permite que o usuário compare os valores físicos sobre planetas obtidos por diferentes métodos de detecção; preconize alvos para novas observações com base em resultados publicados anteriormente; elabore algoritmos que permitam a detecção de planetas não relatados nos estudos originais; desenvolva conhecimentos científicos com estudos sobre outros temas associados, tais como: eclipses binárias; estrelas variáveis; fenômenos dependentes do tempo; atmosferas estelares; rotação das estrelas, manchas estelares; variabilidade estelar intrínseca; comportamentos de progenitores de supernovas, etc. A NASA (sigla em inglês para "National Aeronauticsand Space Administration" ou, em português, Administração

\footnotetext{
${ }^{3}<$ http://exoplanetarchive.ipac.caltech.edu/>.
} 
DOI: https://doi.org/10.33238/ReBECEM.2021.v.5.n.2.26865

Nacional da Aeronáutica e Espaço, apresenta em seu site ${ }^{4}$, informações e dados atualizados e dispostos de modo didático acerca do estudo de exoplanetas.

Outra fonte valiosa de informações sobre o atual estado da arte a respeito do estudo de exoplanetas é o site "exoplanets.org" "que apresenta as compilações com dados e parâmetros produzidos pelo EOD - Exoplanet Orbit Database (HAN et al., 2014), mas que parou de ser atualizado com novos planetas em junho de 2018, segundo informa o próprio site. Os sites do "Hubble Space Telescope" e do "Space Telescope Science Institute" () também apresentam textos e recursos didáticos valiosos (como vídeos explicativos) sobre os conhecimentos científicos consolidados acerca de exoplanetas, como, por exemplo, sobre a formação dos planetas extra-solares e sobre as suas condições de habitabilidade.

Já a "Enciclopédia de Planetas Extra-solares"8 ("The Extrasolar Planets Encyclopaedia") fornece informações e dados a respeito de detecções de exoplanetas obtidas por astrônomos profissionais. Essa é uma ferramenta digital importante para a divulgação científica da exoplanetologia, pois ela avalia as principais técnicas envolvidas para a detecção dos Exoplanetas. Nas técnicas de detecção direta, que apresentam uma dificuldade experimental muito maior, é feita uma análise na intensidade de brilho do planeta; porém, como a quantidade de luz emitida pela estrela é milhares de vezes maior que a porção de luz que um planeta pode refletir, isso é como tentar observar um vagalume ao lado de um farol de sinalização de um barco muito distante de nós. As técnicas de detecção indireta são aquelas que conseguiram detectar o maior número de Exoplanetas catalogados; as principais técnicas utilizadas nessa categoria envolvem o trânsito planetário, a velocidade radial, o pulsar timing (tempo de pulsar), as microlentes gravitacionais e a astrometria (ALMEIDA, 2017). Duas técnicas que mais se destacam por seu sucesso em detectar e conseguir dados referentes a exoplanetas são: o método de trânsito e o método de velocidade radial (TEIXEIRA, 2016).

Grandes telescópios fornecem dados possíveis, não somente para evidenciar a existência de um exoplaneta, mas também, determinar características físicas desse exoplaneta. Dentre os principais telescópios terrestres, vale destacar, por exemplo, o

\footnotetext{
${ }^{4}<$ https://exoplanets.nasa.gov/>.

${ }^{5}<$ http://exoplanets.org/>.

${ }^{6}<$ https://hubblesite.org/science/exoplanets $>$.

${ }^{7}<$ https://www.stsci.edu/hst/about/key-science-themes/exoplanets $>$

${ }^{8}<$ http://exoplanet.eu/>.
} 
DOI: https://doi.org/10.33238/ReBECEM.2021.v.5.n.2.26865

HARPS - "High Accuracy Radial Velocity Planet Searcher", que pertence ao Observatório Europeu do Sul (ESO), e está situado no norte do Chile (na região próxima ao deserto de Atacama) - considerada uma das melhores regiões do planeta para observação do céu noturno devido à baixa incidência de nuvens ao longo do ano todo por isso, uma parte considerável dos grandes telescópios do mundo estão situados no norte do Chile. Os dados científicos consolidados indicam que quase toda estrela do tipo do Sol apresenta pelo menos um planeta orbitando em torno dela (AGUILAR; PULLIAM, 2013). Finalmente o "Telescópio Espacial James Webb"10 (“James Webb Space Telescope" - JWST), que tem o seu lançamento previsto para ocorrer no final do ano de 2021, fornecerá dados que possibilitarão a detecção de oxigênio na atmosfera de exoplanetas (LINCOWSKI; LUSTIG-YAEGER; MEADOWS, 2019).

\section{Exoplanetas no ensino}

A astronomia - em especial o estudo de exoplanetas - pelo seu caráter inerentemente interdisciplinar, pode colaborar com o ensino de conteúdos científicos e motivar novos talentos para áreas profissionais e de pesquisa consideradas áridas por alguns, mas que, na verdade apresentam muitos aspectos fascinantes por diversos pontos de vista. Além disso, ela pode provocar uma reflexão mais ampla dos alunos acerca do mundo em que vivem, capacitando-os para analisar criticamente os resultados adotados pelas políticas públicas para a ciência (CARNEIRO; LONGHINI, 2015).

Surpreendentemente, por mais inovadora que seja a ciência dos exoplanetas, a Física básica envolvida lida com conceitos de mecânica clássica que são bastante acessíveis aos estudantes, para tentar, por exemplo, estimar a massa, o raio e a densidade de um exoplaneta, a partir de dados disponíveis sobre ele (DELLA-ROSE et al., 2018). A equipe da "NASA Kepler Mission Educationand Public Outreach"11 fornece diversas propostas para atividades que podem ser usadas em sala de aula, para alunos de diferentes idades, abordando os métodos de procura por exoplanetas e os conhecimentos científicos envolvidos.

\footnotetext{
${ }^{9}<$ https://www.eso.org/public/teles-instr/lasilla/36/harps/>.

$10<$ https://webbtelescope.org/>.

${ }^{11}<$ https://www.nasa.gov/kepler/education/formal $>$.
} 


\section{O "Royal Observatory Edinburgh" com o "InstituteofPhysics Scotland",} desenvolveram materiais escritos para aulas de Ciências, que podem ser usados no ensino fundamental e no ensino médio, nos quais os alunos investigam exoplanetas, a partir de dados de pesquisas reais. As fichas de dados, as notas para professores e dois vídeos curtos com explicações ("Introductionto Exoplanets" e "Usingthe Deep Space Exoplanets Resources") podem ser acessados pelos interessados ${ }^{12}$. Da mesma forma, a professora CatyPilachowski, da Universidade de Indiana (EUA), produziu roteiros educacionais para aulas introdutórias de astronomia, sobre diferentes aspectos da ciência dos exoplanetas, tais como as zonas de habitabilidade, a fórmula de Drake, o cálculo das massas dos planetas e as estimativas sobre a existência de vida na Via Láctea; esses roteiros estão disponíveis para serem acessados ${ }^{13}$.

A busca por exoplanetas que tenham condições de habitabilidade oferece excelentes oportunidades para que os alunos tenham melhor compreensão, tanto a respeito da natureza da investigação científica, quanto sobre algumas ideias centrais na Física, como, por exemplo, sobre a força da gravidade, as leis do movimento, a interação da luz e da matéria (GOULD; SUNBURY; KRUMHANSL, 2012).

O estudo de exoplanetas, até por ser uma área que está se desenvolvendo na fronteira da ciência atual, permite que os alunos elaborem de início suas próprias hipóteses sobre os sistemas planetários e, durante o processo educacional, revisem os conhecimentos existentes de modo a propor problemas que sejam investigáveis e que estejam relacionados às propriedades relativas de sistemas planetários extra-solares que possam ser determinadas. Uma ênfase no processo de fazer perguntas suscetíveis ao processo de investigação científica é importante no âmbito educacional, pois esta é uma habilidade valiosa em várias situações. As perguntas são as bases da pesquisa científica e componentes cruciais da experiência científica autêntica (MCCONNELL et al., 2012).

Atividades de ensino sobre exoplanetas podem trazer benefícios educacionais e ampliar os conhecimentos científicos apropriados pelos alunos, apresentando um campo de pesquisa contemporâneo em sala de aula e explorando tanto conceitos físicos e astrofísicos importantes - como órbitas planetárias, mecânica celeste, tipos de estrelas e evolução do sistema solar - quanto conceitos que extrapolam a área da Física - como a

\footnotetext{
${ }^{12}<$ https://www.stem.org.uk/resources/elibrary/resource/31030/exoplanets>.

$13<\mathrm{https}$ ://www.astro.indiana.edu/catyp/minilabs.html $>$.
} 


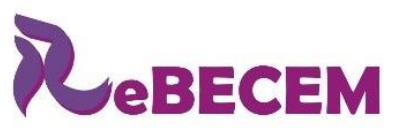

Revista Brasileira de Educação em
Ciências e Educação Matemática

DOI: https://doi.org/10.33238/ReBECEM.2021.v.5.n.2.26865

astrobiologia (estudo da vida no universo), tópicos de estatística, análise de gráficos, teoria de erros, tratamento de dados e tópicos de computação (GEORGE, 2011).

Supondo que uma estrela e seu planeta realizem órbitas circulares em torno do centro de massa do sistema, uma possibilidade didática de trabalho com dados existentes sobre exoplanetas (a partir da técnica da velocidade radial) envolve o uso da terceira lei de Kepler para obter uma expressão inter-relacionando a massa da estrela M, o período orbital T e o raio da órbita $\mathrm{r}$ (aproximando-a como sendo circular). O intervalo de tempo entre o deslocamento máximo para o vermelho e o deslocamento máximo para o azul no espectro da estrela permite determinar o período orbital. A partir do valor da massa da estrela é possível estimar o raio da órbita do exoplaneta (LOPRESTO; MCKAY, 2005). Adicionalmente, com o valor da velocidade orbital, pode-se também calcular o limite mínimo para a massa do planeta.

O uso de imagens em movimento (GIF), disponíveis na internet, pode também ajudar a explicar, de forma visual e didática, as técnicas mais comuns usadas para detecção de exoplanetas, como, por exemplo, o método da velocidade radial ${ }^{14}$ e o método do trânsito fotométrico ${ }^{15}$ (MARCELINO, 2019). Essas imagens em movimento (GIF) podem ser utilizadas com sucesso em atividades educacionais e de divulgação científica acerca de planetas extra-solares.

Experimentos utilizando materiais de baixo custo e facilmente acessíveis, podem também ser bastante úteis para simular técnicas de detecção de exoplanetas. Por exemplo, com o uso de um smartphone como um luxímetro (sensor de iluminância), é possível simular a observação de exoplanetas pelo método de trânsito, ou seja, por meio da detecção da variação da iluminância da estrela hospedeira, mensurando a redução do seu brilho quando um exoplaneta transita na sua frente, percorrendo o seu disco (BARROSO; OLIVEIRA; JESUS, 2020; MARRANGHELLO et al., 2020).

\section{Ações realizadas e discussões}

Esta pesquisa analisa a realização de atividades interdisciplinares de divulgação científica sobre o estudo de exoplanetas que foram realizadas junto a alunos de escolas

\footnotetext{
${ }^{14}<$ http://planeta.rio/wp-content/uploads/2019/07/imagem-2.gif $>$.

$15<$ http://planeta.rio/wp-content/uploads/2019/07/imagem-1.gif $>$.
} 
DOI: https://doi.org/10.33238/ReBECEM.2021.v.5.n.2.26865

de Educação Básica do litoral norte paulista no segundo semestre de 2018. Em particular, foi investigado como metodologias de ensino com essas características permitem alavancar processos de educação científica e podem motivar os jovens para o estudo das ciências naturais. As ações implementadas procuraram considerar o fato de os alunos apresentarem, cada vez mais, novas maneiras de aprender (ANTUNES, 2007), algo que se relaciona intensamente com a multiplicidade de possibilidades de navegação na internet e de recursos tecnológicos à disposição dos cidadãos contemporâneos.

Inicialmente foi feita uma investigação tanto acerca dos conceitos e métodos científicos utilizados no estudo de exoplanetas, quanto dos recursos educacionais existentes na internet para ensinar estes assuntos, com o intuito de planejar e estruturar atividades educacionais de divulgação científica com uso de simulações computacionais, imagens e vídeos que permitissem uma transposição didática satisfatória dos conhecimentos existentes e consolidados atualmente acerca desta área da astrofísica. A utilização de softwares livres, que simulam a detecção de exoplanetas e estão disponíveis gratuitamente na internet, como o "Exoplanet transit hunt" 16 , apresentou um grande potencial educativo como ferramenta didática. As atividades audiovisuais que foram implementadas permitiram articular diferentes metodologias que tornaram mais palpáveis as ideias e conceitos associados ao estudo de exoplanetas.

Nos meses de setembro e outubro de 2018, atividades de divulgação científica sobre exoplanetas foram realizadas pelos autores deste artigo em quatro escolas situadas em municípios do litoral norte paulista: para alunos de ensino médio de duas escolas estaduais e para alunos dos últimos anos do ensino fundamental de duas escolas municipais.

Os alunos presentes nessas atividades de divulgação científica que foram realizadas em diferentes escolas da região eram escolhidos por coordenadores e professores destas instituições, sendo que um dos critérios foi priorizar aqueles que tivessem um maior interesse por temas científicos. Tal método de escolha gerou uma reflexão sobre se a atividade deveria ser aplicada aos alunos que já estavam mais motivados para aprender acerca de tópicos científicos ou a quaisquer alunos, independentemente de seus interesses prévios sobre ciência. Porém, como os temas associados ao estudo de exoplanetas empolgavam alguns alunos, mas seguramente não todos os alunos, como havia um limite máximo de 40 alunos que poderiam participar em

\footnotetext{
${ }^{16}<$ http://www.planetarium-activities.org/shows/sp/exoplanet-transit-hunt $>$.
} 


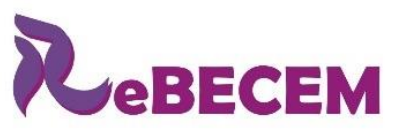

\section{Revista Brasileira de Educação em \\ Ciências e Educação Matemática}

DOI: https://doi.org/10.33238/ReBECEM.2021.v.5.n.2.26865

cada atividade (limite este condicionado tanto pela natureza das ações planejadas, quanto pelas características dos espaços físicos onde estas atividades foram realizadas) e como os ambientes escolares eram estranhos aos autores deste trabalho (que estavam visitandoos na condição de convidados dos profissionais de cada instituição escolar em específico) e devido à necessidade de controle mínimo sobre as atitudes e o comportamento dos jovens estudantes presentes, para poder implementar as atividades a contento, o critério adotado pelos gestores e professores da escola para selecionar os alunos presentes foi o de conveniência.

O tipo de público presente com certeza influenciou os resultados educacionais obtidos com as ações. Um exemplo, foi o de alunos de uma escola municipal de Caraguatatuba, selecionados entre aqueles que participavam de um projeto mais avançado de matemática daquela escola, inclusive com vários alunos tendo participado dos minicursos de astronomia realizados anualmente no campus de Caraguatatuba do Instituto Federal de São Paulo (IFSP) entre 2015 e 2019.

Metodologicamente, este é um trabalho do tipo pesquisa-ação ou de pesquisa participante, pois os pesquisadores e alunos interagiram no trabalho de pesquisa de um modo colaborativo, o que propiciou proximidade com o meio social e realidade local das escolas nas quais as ações ocorreram; a análise dos resultados teve um caráter qualitativo, como acontece com muitos outros trabalhos de pesquisa na área da divulgação científica, com o intuito de investigar diferentes aspectos do processo de aprendizagem acerca dos temas tratados (OLIVEIRA, 2015). O escopo desta investigação abordou ao mesmo tempo, a divulgação científica e o ensino de ciências, pois as atividades de divulgação científica foram trabalhadas pelos autores na interface com escolas de educação básica (IANINI et al., 2007).

Os resultados das atividades implementadas foram analisados a partir das observações realizadas pelos dois autores sobre as reações dos alunos, tanto acerca dos conteúdos científicos apresentados, quanto dos recursos didáticos utilizados para explicar os métodos de detecção de exoplanetas. Em particular, as dúvidas que surgiram entre os estudantes e os questionamentos que eles fizeram durante as apresentações foram analisados com o intuito de entender os obstáculos que apareceram no processo de aprendizagem e os interesses revelados pelos alunos acerca dos temas tratados.

Durante as atividades de divulgação científica realizadas na forma de palestras mediadas pelo uso de recursos audiovisuais, com duração de cerca de 30 minutos, notouse junto aos alunos um expressivo interesse por tópicos científicos relacionados à 
DOI: https://doi.org/10.33238/ReBECEM.2021.v.5.n.2.26865

astronomia. O uso de imagens e vídeos nessas atividades permitiu tornar os conhecimentos mais tangíveis e concretos aos alunos. Em uma das escolas de ensino médio em que foram realizadas atividades de divulgação científica, foi possível notar, pelos cartazes existentes na sala de vídeo, que os alunos haviam recentemente feito uma pesquisa para um projeto de estudo sobre a possibilidade de colonização em Marte no futuro. Este projeto, que fez com que os alunos se aprofundassem sobre assuntos relacionados à astronomia, permitiu que durante a ação os estudantes fizessem perguntas com maior embasamento e adquirissem melhor compreensão acerca dos conceitos apresentados sobre exoplanetas.

Algo que ficou nítido pelas apresentações realizadas é que a ciência dos exoplanetas é bastante interdisciplinar, o que torna possível a sua utilização para contribuir no ensino de diversas disciplinas curriculares como a matemática (em associação com o estudo de conceitos algébricos e na elaboração de gráficos), a biologia (sobretudo no ramo da exobiologia ou astrobiologia, área que investiga sobre a formação e a distribuição de vida no espaço) e a química (no que diz respeito à composição química de cada planeta e da sua atmosfera, o que tem um grande impacto para o estudo da probabilidade de existência de vida).

Trabalhos anteriores, sobre a mesma temática que esta, mostram tanto que os conceitos básicos de física necessários para uma compreensão dos métodos de detecção de exoplanetas são geralmente abordados em cursos introdutórios de física, como é o caso do centro de massa e da conservação de momento linear (DELLA-ROSE et al., 2018), quanto que atividades sobre exoplanetas são bons exemplos práticos de astronomia para serem usados em sala de aula, já que permitem tratar de forma interdisciplinar de diversos tópicos relevantes para a formação científicados alunos em diferentes áreas do conhecimento (GEORGE, 2011).

Dentre os temas que acabaram sendo levantados pelos alunos participantes nas atividades, dois deles se destacaram: a possibilidade de existência de vida fora da Terra e a possibilidade de existência de vida inteligente fora da Terra. Nesses momentos, era explicado que as duas questões são bem diferentes, pois, pelo menos na Terra, a vida é algo bastante presente, mas, quanto à vida inteligente, ocorre o contrário. Mesmo que esses tópicos não guardem uma relação mais estreita com a temática dos exoplanetas é importante aproveitar o interesse dos alunos por essas questões para aprofundar discussões a respeito dos métodos que a ciência utiliza para produzir conhecimentos. 
DOI: https://doi.org/10.33238/ReBECEM.2021.v.5.n.2.26865

Durante as ações, os estudantes foram indagados com a pergunta: "Você acha que existe vida inteligente fora da Terra?" Dentre aqueles que responderam afirmativamente, destacaram-se os seguintes depoimentos: "Sim, pois eu acho que existem muitos outros sistemas e planetas"; "Pelo meu conhecimento, eu acho que pode existir, porque o espaço é infinito"; "O universo é enorme, com muitos sistemas, deve existir"; "Nós somos a prova viva disso e existem milhares de outros planetas"; "Porque não é possível que isto só ocorra na Terra, com um universo tão vasto". Dentre as respostas negativas, destacaram-se os seguintes depoimentos: "Porque acho que somos os únicos seres vivos com raciocínio"; "Não existem dados suficientes que provam o contrário"; "Pois é muito difícil existir vida inteligente"; "Porque já foi difícil ter vida inteligente na Terra com as condições que temos e acho difícil ter mais planetas com essas condições”.

Dentre os temas abordados nas ações educacionais implementadas durante esta pesquisa estiveram: o surgimento de sistemas planetários, o modo pelo qual os planetas se distribuem em torno de estrelas, a zona de habitabilidade em torno de uma estrela, os métodos de detecção de exoplanetas e as dificuldades existentes no campo da ciência e da tecnologia para a detecção de exoplanetas. Os resultados obtidos por este trabalho permitiram avaliar que há um leque amplo de potencialidades didáticas para o trabalho educacional sobre o tema dos exoplanetas na educação básica.

\section{Considerações finais}

Esta pesquisa analisou a elaboração e a realização de atividades de divulgação científica que tiveram o estudo de exoplanetas como seu eixo básico, com o objetivo tanto de auxiliar no ensino de tópicos de Física, quanto de motivar os jovens para explorarem e se aprofundarem nos conhecimentos científicos envolvidos neste estudo.

O trabalho desenvolvido percebeu ser importante estimular os alunos a adotarem formas de pensar científicas na formulação e no teste de hipótese, o que pode conferir autonomia aos alunos e produzir um aumento da autoconfiança à medida que eles passam a dominar e se apropriar dos conceitos científicos existentes, uma habilidade de trabalho importante em diversas carreiras profissionais da atualidade. Este trabalho possibilitou observar como, de fato, atividades em sala de aula sobre temas da astronomia permitem que o trabalho dialogue com o cotidiano e as expectativas de muitos estudantes, bem como, proporcionam ricas discussões sobre as interrelações da ciência e da tecnologia 
DOI: https://doi.org/10.33238/ReBECEM.2021.v.5.n.2.26865

com a sociedade o que pode tornar o ensino menos fragmentado e mais contextualizado (SIEMSEN; LORENZETTI, 2020).

As atividades realizadas foram projetadas com a intenção de ensinar simultaneamente habilidades acerca de processos científicos e conteúdos sobre exoplanetas e métodos de detecção. O uso da história acerca do estudo de exoplanetas como um dos fios condutores das atividades realizadas ajudou a promover reflexões pelos alunos sobre como a ciência é um processo contínuo de construção originário do esforço coletivo da humanidade. Finalmente, as atividades revelaram a existência de um grande interesse dos jovens por temas de astronomia, em especial pelo estudo de exoplanetas, o que pode ser usado por professores de disciplinas científicas da educação básica, como é o caso da Física.

\section{Agradecimentos}

Agradecemos ao CNPq pela bolsa de iniciação científica concedida a Ryan Nepomuceno Montemor, um dos autores deste artigo.

\section{Referências}

AGUilar, D. A.; PUlliaM; C. At Least One in Six Stars Has an Earth-sized Planet. Center for Astrophysics, 2013. Disponível em: 〈https://www.cfa.harvard.edu/news/2013-01〉. Acesso em: 5 jan. 2021.

AKESON, R. L. The NASA Exoplanet Archive: Data and Tools for Exoplanet Research. Publications of the Astronomical Society of the Pacific, s/l, v. 125, p. 989-999, August 2013. Disponível em: <https://iopscience.iop.org/article/10.1086/672273/pdf>. Acesso em: 3 jan. 2021.

ALMEIDA, L. de. Estudo da topologia de microlentes gravitacionais e a descoberta de exoplanetas do tipo Terra na zona habitável. 2017. 101 f. Dissertação (Mestrado em Física) Departamento de Física Teórica e Experimental, Universidade Federal do Rio Grande do Norte (UFRN), Natal, RN, 2017. Disponível em:

<https://repositorio.ufrn.br/bitstream/123456789/23146/1/LeandroDeAlmeida_DISSERT.pdf>. Acesso em: 24 jul. 2021.

AMORIM, R. G. G.; SANTOS, W. C. Calculation of mass and orbital data of exoplanets by Doppler method. Revista Brasileira de Ensino de Física [online], v. 39, n. 1, e1310, 2017. 
DOI: https://doi.org/10.33238/ReBECEM.2021.v.5.n.2.26865

ANDRADE, M. H. de. Exoplanetas como tópico de Astronomia motivador e inovador para o ensino de Física no ensino médio. 2012. 126 f. Dissertação (Mestrado Profissional em Ensino de Física) - Instituto de Física, Universidade Federal do Rio Grande do Sul, Porto Alegre, RS, 2012. Disponível em:

<https://lume.ufrgs.br/bitstream/handle/10183/70396/000877205.pdf?sequence=1\&isAllowed= y>. Acesso em: 24 jul. 2021.

ANTUNES, C. Novas maneiras de ensinar, novas formas de aprender. Porto Alegre: Artmed, 2007.

BANKS; T.; RHODES; M. D.; BUDDING, E. An Update on the Student Exoplanet Programme. ArXiv, 2020. Disponível em:

<https://arxiv.org/ftp/arxiv/papers/2006/2006.09552.pdf>. Acesso em: 6 jan. 2021.

BARROSO, R. R.; OLIVEIRA, A. L. de; JESUS, V. L. de. Simulação da detecção de exoplanetas pelo método do trânsito utilizando o pêndulo cônico e o smartphone. Revista Brasileira de Ensino de Física [online], v. 42, e20200161, 2020. Disponível em: <https://doi.org/10.1590/1806-9126-rbef-2020-0161 >. Acesso em: 6 jan. 2021.

BRUNO, G. Sobre o infinito, o universo e os mundos: Os pensadores - Buno, Galileu, Campanella. São Paulo: Abril Cultural, 1978.

CARNEIRO, D. L. C. M.; LONGHINI, M. D. Divulgação científica: as representações sociais de pesquisadores brasileiros que atuam no campo da Astronomia. Revista Latino-Americana de Educação em Astronomia - RELEA, São Carlos, n. 20, p. 7-35, 2015.

DELLA-ROSE, D.; CARLSON, R.; HARPE, K. de L.; NOVOTNY, S.; POLSGROVE, D. Exoplanet Science in the Classroom: Learning Activities for an Introductory Physics Course. The Physics Teacher, s/l, v. 56, p. 170-173, 2018.

FISCHER, D.; LUNINE, J.L.; HAMMEL, H.; HENNING, T.; HILLENBRAND, L.; KASTING, J.; LAUGHLIN, G.; MACINTOSH, B.; MARLEY, M.; MELNICK, G.; MONET, D.; NOECKER, C.; PEALE, S.; QUIRRENBACH, A.; SEAGER, S.; WINN, J. Worlds

Beyond: A Strategy for the Detection and Characterization of Exoplanets. ArXiv, 2010. Disponível em: <https://arxiv.org/pdf/0808.2754.pdf>. Acesso em: 5 jan. 2021.

GEORGE, S. J. Extrasolar Planets in the Classroom. ArXiv, 2011. Disponível em: <https://arxiv.org/pdf/1103.5690.pdf>. Acesso em: 3 jan. 2021.

GOULD, R. R.; SUNBURY, S.; KRUMHANSL, R. Using online telescopes to explore exoplanets from the physics classroom. American Journal of Physics, San Francisco, v. 80, n. 5, p. $445-451,2012$.

HAN, E.; WANG, S. X.; WRIGHT, J. T.; FENG, Y. K.; ZHAO, M.; FAKHOURI, O.; BROWN, J. I.; HANCOCK, C. Exoplanet Orbit Database. II. Updates to Exoplanets.org. 
DOI: https://doi.org/10.33238/ReBECEM.2021.v.5.n.2.26865

Publications of the Astronomical Society of the Pacific, s/l, v. 126, p. 827-837, September 2014. Disponível em: 〈https://iopscience.iop.org/article/10.1086/678447/pdf〉. Acesso em: 2 jan. 2021.

IANINI, A. M. N.; FARES, D. C.; BIZERRA, A.; MARANDINO, M. Pesquisa em divulgação científica: um levantamento de referenciais teóricos nacionais. In: ENCONTRO NACIONAL DE PESQUISA EM EDUCAÇÃO EM CIÊNCIAS, 6, 2007, Florianópolis. Resumos do VI Encontro Nacional de Pesquisa em Educação em Ciências (ENPEC)... Florianópolis: ABRAPEC, 2007, p. 1-12. Disponível em: <http://www.geenf.fe.usp.br/v2/wpcontent/uploads/2012/10/Divulg_cient_teorico_2007.pdf>. Acesso em: 20 mai. 2021.

LINCOWSKI, A. P.; LUSTIG-YAEGER, J.; MEADOWS, V. S. Observing Isotopologue Bands in Terrestrial Exoplanet Atmospheres with the James Webb Space Telescope: Implications for Identifying Past Atmospheric and Ocean Loss. The Astronomical Journal, s/l, v. 158, n. 1, p. 1-26, 2019.

LOPRESTO, M. C.; MCKAY, R. An introductory physics exercise using real extrasolar planet data. Physics Education, s/l, v. 40, n. 1, p. 46-50, 2005.

MANDELL, M. Physics experiments for children. New York: Dover Publications, 2013.

MARCELINO, J. Observação direta de Exoplanetas. 2019. Disponível em: <http://planeta.rio/observacao-direta-de-exoplanetas/>. Acesso em: 27 jan. 2021.

MARRANGHELLO, G. F.; FALCÃO, J. V.; AVEIRO, T. L. B.; LUCAS, W. M. O uso do smartphone para a simulação do método de trânsito em sala de aula. Física na Escola, s/l, v. 18, n. 1, p. 9-13, 2020. Disponível em: <http://www1.fisica.org.br/fne/phocadownload/Vol18Num1/FnE-18-1-190505.pdf>. Acesso em: 6 jan. 2021.

MAYOR, M.; QUELOZ, D. A Jupiter-mass companion to a solar-type star. Nature, s/1, v. 378, n. 6555, p. 355-359, 1995.

MCCONNELL, N. J.; MEDLING, A. M.; STRUBBE, L. E.; MOTH, P.; MONTGOMERY, R. M.; RASCHKE, L. M.; HUNTER, L.; GOZA, B. A College-Level Inquiry-Based Laboratory Activity on Transiting Planets. ArXiv, 2012. Disponível em: <https://arxiv.org/pdf/1009.3940.pdf>. Acesso em 2 jan. 2021.

NASA. Life n Earth ... and elsewhere? Astrobiology in your classroom. 2006. Disponível em: $<$ https://astrobiology.nasa.gov/nai/media/medialibrary/2013/10/Astrobiology-Educator-Guide2007.pdf>. Acesso em: 20 mai. 2021.

OLIVEIRA, G. L. de. Panorama das pesquisas sobre divulgação científica / popularização da ciência no Brasil. 2015. 98 f. Dissertação (Mestrado em Educação em Ciências) Universidade Federal do Rio Grande (FURG), Rio Grande, RS, 2015. Disponível em: 
DOI: https://doi.org/10.33238/ReBECEM.2021.v.5.n.2.26865

<https://sistemas.furg.br/sistemas/sab/arquivos/bdtd/0000010810.pdf>. Acesso em: 20 mai. 2021.

PERRYMAN, M. The Exoplanet Handbook. 2. ed. Cambridge, UK: Cambridge University Press, 2018.

SCHNEIDER, J.; DEDIEU, C.; SIDANER, P. L.; SAVALLE, R.; ZOLOTUKHIN, I. Defining and cataloging exoplanets: the exoplanet.eu database. Astronomy \& Astrophysics, s/1, v. 532, A79, p. 1-13, 2011. Disponível em: <https:/www.aanda.org/articles/aa/pdf/2011/08/aa1671311.pdf>. Acesso em: 3 jan. 2021.

SIEMSEN, G. H.; LORENZETTI, L. O ensino de astronomia e a alfabetização científica e tecnológica: uma abordagem no ensino médio. Revista Areté - Revista Amazônica de Ensino de Ciências, Manaus, v. 14, n. 28, p. 137-151, 2020. Disponível em:

<http://periodicos.uea.edu.br/index.php/arete/article/view/1991>. Acesso em: 28 jan. 2021.

STRUVE, O. Proposal for a project of high-precision stellar radial velocity work. The Observatory (Provided by the NASA Astrophysics Data System), s/1, v. 72, p. 199-200, 1952.

TEIXEIRA, M. A. Ferramentas da astroestatística para o estudo da velocidade radial estelar. 2016. 79 f. Dissertação (Mestrado em Física) - Departamento de Física Teórica e Experimental, Universidade Federal do Rio Grande do Norte (UFRN), Natal, RN, 2016. Disponível em:

<https://repositorio.ufrn.br/bitstream/123456789/21587/1/MarcioAssuncaoTeixeira_DISSERT. pdf>. Acesso em: 24 jul. 2021.

WOLSZCZAN, A.; FRAIL, D. A. A planetary system around the millisecond pulsar PSR1257 + 12. Nature, s/1, v. 355, p. 145-147, 1992.

Recebido em: 31 de janeiro de 2021

Aceito em: 12 de junho de 2021 